148 ANTIMALARIAL AGENTS AND OTHER
IMMUNOSUPPRESSANTS INFLUENCE BAFF LEVELS IN
OPPOSITE DIRECTIONS IN PATIENTS WITH SYSTEMIC
LUPUS ERYTHEMATOSUS

'Borja Hernandez-Breijo, ${ }^{2}$ Alvaro Gomez, ${ }^{2}$ Petter Johansson, ${ }^{2}$ Sofia Soukka, ${ }^{2}$ Ioannis Parodis*. 'Immuno-Rheumatology Research Group, IdiPaz, La Paz University Hospital; ${ }^{2}$ Department of Medicine, Division of Rheumatology, Karolinska Institutet

10.1136/lupus-2019-Ism.148

Background Elevated levels of the B-cell activating cytokine BAFF (also known as BLyS) have been associated with active systemic lupus erythematosus (SLE), and methotrexate use has been shown to increase soluble BAFF levels. The anti-BAFF monoclonal antibody belimumab has been approved as an add-on to standard-of-care SLE treatment, mainly comprising glucocorticoids, antimalarial agents (AMA) and other immosuppressants. We aimed at investigating the effect of AMA and three other commonly used immunosuppressants in SLE (methotrexate, azathioprine, mycophenolic acid) on serum BAFF levels.

Methods We analysed data from two phase III clinical trials of belimumab, the BLISS-52 $(\mathrm{n}=865$; NCT00424476) and BLISS-76 ( $\mathrm{n}=819$; NCT00410384) trials. Access to data was granted by GlaxoSmithKline. Baseline serum samples (before belimumab initiation) were obtained from the patients and stored at $-80^{\circ} \mathrm{C}$ until BAFF level determination using ELISA. The Mann-Whitney $U$ test was used to compare BAFF level distributions between treatment groups. Subsequently, linear regression models were applied to determine independence.

Results BAFF levels were higher in patients receiving methotrexate (mean, SD: 1835, $1617 \mathrm{pg} / \mathrm{mL} ; \mathrm{n}=212 ; \mathrm{p}=0.001$ ), azathioprine (mean, SD: 1901, $1472 \mathrm{pg} / \mathrm{mL} ; \mathrm{n}=364$; $\mathrm{p}<0.001$ ) and mycophenolic acid (mean, SD: 1994, $1544 \mathrm{pg} /$ $\mathrm{mL} ; \mathrm{n}=175 ; \mathrm{p}<0.001)$ and no immunosuppressant other than the one investigated (AMA allowed) compared with patients receiving no immunosuppressive treatment other than AMA (mean, SD: $1593 \pm 1929 ; \mathrm{n}=860$ ). In contrast, patients on AMA displayed lower BAFF levels (mean, SD: 1654, $1318 \mathrm{pg} /$ $\mathrm{mL} ; \mathrm{n}=1085$ ) compared with patients who did not use AMA (mean, SD: 1942, $2408 \mathrm{pg} / \mathrm{mL} ; \mathrm{n}=580 ; \mathrm{p}=0.002$ ). In linear regression, AMA use showed a consistent and independent association with lower BAFF levels in all models, whereas use of each one of methotrexate, azathioprine and mycophenolic acid showed associations with higher BAFF levels. Each one of the models were adjusted for the use of immunosuppressants other than the one investigated.

Conclusions We observed a differential effect of antimalarial agents and other immunosuppressants on BAFF levels, reflecting the different mechanisms of action of these drugs. Considering the importance of BAFF levels in B-cell homeostasis and the pathogenesis of SLE, these findings should be taken into account in the therapeutic management of SLE and the concomitant administration of different treatments, including BAFF inhibitors.

Funding Source(s): The study was supported by grants from the Swedish Research Council, Professor Nanna Svartz Foundation (2017-00213), Swedish Rheumatism Association, King Gustaf Vs 80 year Foundation, Ingegerd Johanssons Fund, Stockholm County Council and Karolinska Institutet Foundations.

\section{9 NETWORK-BASED ANALYSIS OF CLINICAL AND MOLECULAR DATA IN A MULTIETHNIC LUPUS COHORT IDENTIFIES MOLECULAR ASSOCIATIONS WITH SEROLOGICAL MANIFESTATIONS}

${ }^{1}$ Ishan Paranjpe* ${ }^{2}$ Cristina Lanata, ${ }^{2}$ Joanne Nititham, ${ }^{2}$ Kimberly Taylor, ${ }^{2}$ Milena A Gianfrancesco, ${ }^{3}$ Manish Paranjpe, ${ }^{4}$ Shan Andrews, ${ }^{2}$ Sharon Chung, ${ }^{5}$ Brooke Rhead, ${ }^{5}$ Lisa Barcellos, ${ }^{6}$ Laura Trupin, ${ }^{3}$ Patricia Katz, ${ }^{6}$ Maria C DallEra, ${ }^{6}$ Jinoos Yazdany, ${ }^{4}$ Marina Sirota, ${ }^{2}$ Lindsey Criswell. ${ }^{1}$ Icahn School of Medicine at Mount Sinai; ${ }^{2}$ Division of Rheumatology, Department of Medicine, University of California, San Francisco; ${ }^{3}$ UCSF; ${ }^{4}$ Baker Institute for Computational Health Sciences, University of California, San Francisco; ${ }^{5}$ UC Berkeley; ${ }^{6}$ UC San Francisco

10.1136/lupus-2019-Ism.149

Background Systemic Lupus Erythematous (SLE) is a chronic autoimmune disease with heterogeneous disease manifestations and outcomes. Previous work has found associations between DNA methylation at specific CpG sites and lupus nephritis, serologies, and SLEDAI score. However, these methods examine single CpGs and do not capture the full biological complexity. Using an integrative network-based approach, we aim to define how DNA methylation and genetic variation underlie this clinical heterogeneity in a well-phenotyped multiethnic cohort of SLE patients.

Methods 333 SLE participants from diverse ethnic backgrounds were recruited as part of this study. From peripheral blood, DNA methylation was measured using the Illumina EPIC Beadchip and single nucleotide polymorphism (SNP) genotype data was generated on the Affymetrix LAT1 World Array. Weighted gene correlation network analysis (WGCNA) was applied to the DNA methylation data. The resulting CpG networks were associated with relevant SLE clinical features in a multivariate linear regression model adjusting for population stratification, cell composition, sex, smoking history, medications.

Results We identified one WGCNA CpG module significantly associated with SLEDAI score, anti-Sm, and anti-dsDNA serologies $(\mathrm{FDR}<0.05)$. This network consisted of $303 \mathrm{CpGs}$ and was hypomethylated in patients with higher SLEDAI scores and positive anti-Sm and anti-dsDNA serologies. Pathway analysis of this module revealed significant enrichment of genes in the Type I interferon pathway. We also performed a cismeQTL analysis to determine whether any of the network CpGs were under genetic control. Of the $303 \mathrm{CpGs}$ in the network, $54 \mathrm{CpGs}$ were under proximal genetic control $($ FDR $<0.01)$, suggesting that specific genetic variants play a role in epigenetic regulation of interferon related gene expression in the context of SLE autoantibody production.

Conclusions Overall, we performed a network-based analysis of DNA methylation and identified a network of CpGs significantly associated with SLE serologies and SLEDAI score. Our approach identifies large-scale epigenetic remodeling that drives SLE pathology rather than single CpG associations as in previous studies that may be influenced by stochastic variation. By applying an integrative computational approach, our method serves to reveal the epigenetic and genetic role of the Type I interferon pathway in SLE.

Funding Source(s): This study was funded through the following grants: P30 AR070155 (MS, IP, LC), P60AR053308 (LC), K01LM012381 (MS), F32 AR070585 NIAMS (MG), U01DP005120 CDC (LC, CL, JY, MD, LT, PK), the Rheumatology Research Foundation 128849A (CL), and the Lupus Research Alliance (LC). 


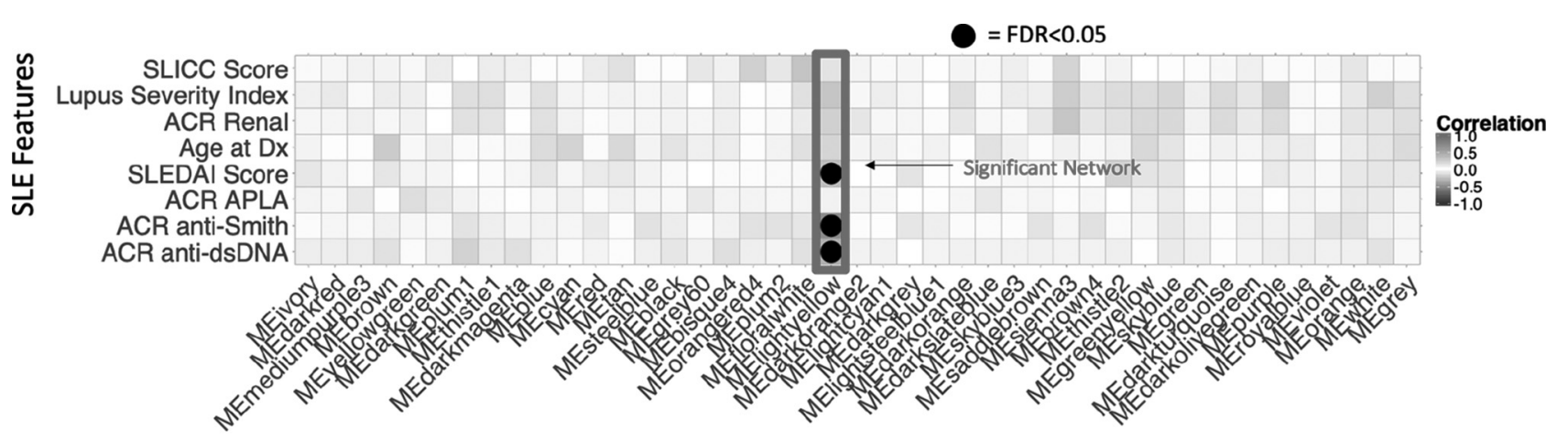

WGCNA Networks
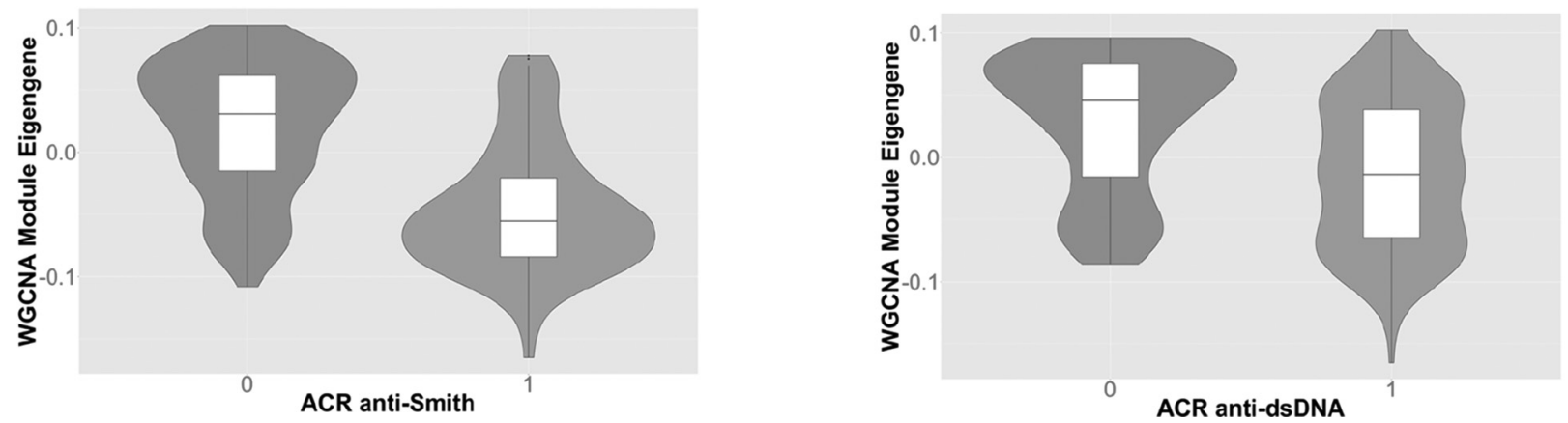

Abstract 149 Figure 1 Associations between WGCNA CpG network and SLE features. WGCNA was applied to methylation data and associated networks with relevant SLE clinical criteria. Top: Colors in squares represent Pearson correlations between individual phenotypes and module eigengenes with significant associations $(F D R<0.05)$ represented by a black circle. Bottom: Distribution of WGCNA eigengene for the identified network shows hypomethylation in patients with anti-Sm and anti-dsDNA serologies.

\section{ANTIMALARIAL AGENTS IMPROVE PHYSICAL FUNCTIONING IN PATIENTS WITH SYSTEMIC LUPUS ERYTHEMATOSUS}

Ioannis Parodis*, Sofia Soukka, Alvaro Gomez, Petter Johansson, Sharzad Emamikia, Yvonne Enman, Katerina Chatzidionysiou. Department of Medicine, Division of Rheumatology, Karolinska Institutet

\subsection{6/lupus-2019-Ism.150}

Background Patients with systemic lupus erythematosus (SLE) suffer an impaired health-related quality of life (HRQoL), and the majority of them experience fatigue as a major problem. Traditionally, treatment of SLE has been symptomatic, and antimalarial agents (AMA) are considered a cornerstone of SLE treatment. In previous literature, results regarding the effect of antimalarial agents on HRQoL have been conflicting. In this study, we aimed at investigating the potential influence of AMA on SLE patients self-perception of HRQoL aspects.

Methods We utilised pooled baseline data from the BLISS-52 and BLISS-76 clinical trials of belimumab $(n=1684)$. Access to data was granted by GlaxoSmithKline. The patients HRQoL and fatigue were self-reported using the Medical Outcomes Study (MOS) short form 36 (SF-36) health survey, the functional assessment of chronic illness therapy (FACIT)-Fatigue scale and the three-level EuroQol- 5 Dimension (EQ-5D) questionnaire. The non-parametric Mann-Whitney $U$ test was used for comparisons between AMA users and non-users. Linear regression models were next used in order to adjust for possible confounding factors; these included age, sex, ethnic origin, SLE disease activity, SLE duration, organ damage, corticosteroid use and use of other immunosuppressants.

Results Patients receiving AMA performed better than patients who did not receive AMA with regard to SF-36 physical component summary (PCS) scores $(\mathrm{p}=0.001)$, physical functioning $(p<0.001)$, role physical $(p=0.036)$, bodily pain $(p=0.016)$, FACIT-Fatigue scores $(p=0.046)$, EQ-5D score $(p=0.004)$ and EQ-5D visual analogue scale (VAS) scores $(p=0.001)$. However, only the difference regarding SF-36 physical functioning was found to be greater than the minimal clinically important difference (MCID) among all SF-36 and FACIT-Fatigue parameters (2.5 points for physical functioning). No MCID for EQ-5D scores or EQ-5D VAS scores has been validated. The observed association regarding physical functioning was still significant after adjustment for confounding factors. In this analysis, Asian patients reported better physical functioning while African/African American patients performed worse. High disease activity and organ damage were also independent factors of worse physical functioning, whereas corticosteroid use independently improved the outcome.

Conclusions AMA use contributes to better physical functioning in patients with SLE, independently of other factors.

Funding Source(s): The study was supported by grants from the Swedish Research Council, Professor Nanna Svartz Foundation (2017-00213), Swedish Rheumatism Association, King Gustaf Vs 80 year Foundation, Ingegerd Johanssons Fund, Stockholm County Council and Karolinska Institutet Foundations. 